\title{
Volmer-Weber Growth of CdTe on Silicon: A One-Dimension Monte Carlo Model
}

\author{
S. O. Ferreira* and S. C. Ferreira Jr. ${ }^{\dagger}$ \\ *Departamento de Física, Universidade Federal de Viçosa, 36571-000, Viçosa, MG, Brazil and \\ † Laboratório de Física Teórica e Computacional, Departamento de Física, \\ Universidade Federal de Pernambuco, 50670-901, Recife, PE, Brazil
}

Received on 4 April, 2005

\begin{abstract}
In the last few years intense efforts have been devoted to the growth and characterization of semiconductor nanostrucutures. In particular, quantum dots of CdTe grown by hot wall epitaxy on $\mathrm{Si}(111)$ substrates constitute a very interesting example. CdTe/Si systems follow the Volmer-Weber growth mode with nucleation of 3D CdTe islands on the Si substrate even for less than one monolayer of evaporated material. In the present work, we proposed a simple one-dimension model to reproduce a very peculiar behavior observed in the quantum dot height and size distributions at distinct temperatures. The model, which includes CdTe deposition, diffusion, and revaporization, qualitatively reproduces these distributions. Moreover, the width distributions suggest a transition from Stranski-Krastanow growth mode at lower temperatures to Volmer-Weber growth mode at higher temperatures.
\end{abstract}

Keywords: Quantum dots of CdTe; Si(111); Volmer-Weber growth

\section{INTRODUCTION}

Semiconductor quantum dot structures have atracted a lot of attention in recent years due to their potential technological applications. The greater part of the processes used to grow these structures are governed by the transition from twoto three-dimensional growth regime driven by the strain energy accumulated in the epitaxial layer [1-7], the StranskiKrastanow (SK) growth mode. Ferreira et. al. considered the growth of CdTe quantum dots (QDs) on Si(111) substrates using hot wall epitaxy (HWE) technique. It was observed that the system follows the Volmer-Weber (VW) growth mode, with nucleation of isolated CdTe 3D islands on the Si even for just 0.6 monolayers (ML) of deposited material [8].

Ferreira et. al. [8] investigated CdTe samples grown on $\mathrm{Si}(111)$ with distinct growth times and substrate temperatures. The effects of temperature can be observed in Fig. 1, in which three AFM images of samples with 1.6 ML of evaporated material grown at (a) 200 , (b) 250 , and (c) $300{ }^{\circ} \mathrm{C}$ are shown. One can see an increase of the QD numbers accompanied with a decreasing of the QD sizes as temperature increases. These results show a very peculiar behavior of mean dot density and size: the first one increases while the second one decreases as a function of increasing growth temperature. Both quantities have the opposite behavior from that observed for selfassembled dots grown under the SK mode [9-11].
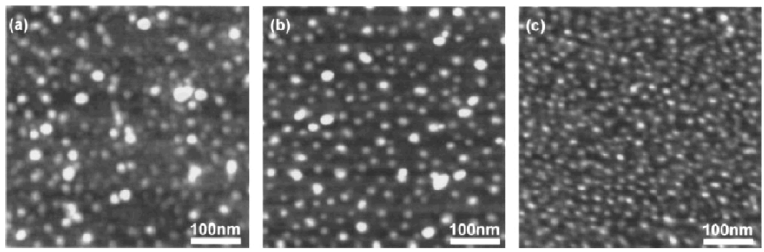

FIG.1. Effects of temperature in the QD structures. Samples with 1.6 ML were grown at (a) 250 , (b) 250 and (c) $300{ }^{\circ} \mathrm{C}$.

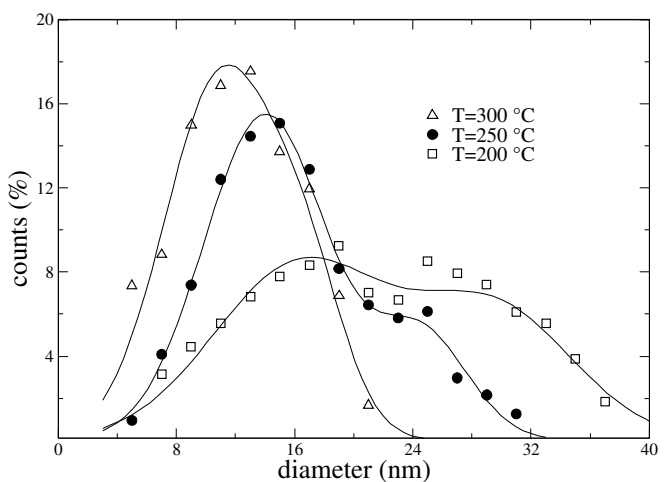

FIG.2. Quantum dot size distributions for the samples shown in Fig. 1. The solid lines correspond to two-peak Gaussian fits.

The QD size distributions for the samples shown in Fig. 1 are drawn in Fig. 2. Independently of temperature, all samples show a bimodal distribution for the QD diameters which can be fitted by two-peak Gaussian functions (solid lines). This bimodal distribution is commonly observed in many systems undergoing SK growth mode [3, 9]. It is important to mention that similar distribution behaviors are found for the QD heights. As one can see, as the growth temperature increases the curves shift to smaller diameters meaning that the number of small dots increases while the number of large ones decreases. According to this work, the main difference between SK and VW growth modes lies in this behavior of the dot size and density distribution as functions of temperature.

In our knowledge, this property of CdTe QD size distributions was reported by the first time in reference [8] and the layer mechanisms related to problem are not well understood . Therefore, this leads us to proposed a model for the deposition of CdTe on $\mathrm{Si}(111)$ including CdTe diffusion and revaporization. The main features of the model are the different rules for diffusion and revaporization of $\mathrm{CdTe}$ on the initial $\mathrm{Si}$ substrate and CdTe on previously deposited CdTe layers. 
The paper layout is the following. Section 2 describes the model and the procedures used in the simulations. In section 3 , the simulational results are presented and discussed in the light of the experiments. Finally, some conclusions are drawn in section 4

\section{MODEL}

The $\operatorname{Si}(111)$ substrate is represented by a one-dimensional lattice with $L$ sites and periodic boundary conditions. The $\mathrm{Si}$ adatoms are not able to diffuse or evaporate while three processes are considered for CdTe: deposition, diffusion and revaporization. The substrate height at the site $j$, i.e., the number of CdTe adatoms on this site, is represented by $h_{j}$.

1. Deposition. CdTe adatoms are deposited on the substrate at a constant rate $R_{1}$. In the deposition, a site is chosen at random and its height is increased by a lattice unity.

2. Diffusion. The diffusion of CdTe is selected (but not necessarily implemented) at a rate $R_{2}$ given by a Arrhenius-like expression

$$
R_{2}=\frac{d^{*} k_{B} T}{h} \exp \left(-E_{d i f} / k_{B} T\right)
$$

where $d^{*}$ is substrate dimension and $E_{\text {dif }}$ is the effective energy for the diffusion activation. The value of $E_{d i f}$ is of the order of $1 \mathrm{eV}$. However, the diffusion can be implemented or not with probabilities which depend on the local configuration. For each tentative, a particle chosen at random hops from its position $j$ to one of its neighbor $j^{\prime}$ with probability

$$
P_{h o p}=\left\{\begin{array}{c}
p^{n+\Delta h} \text { if } \Delta h>0 \\
p^{n} \text { if } \Delta h \leq 0,
\end{array}\right.
$$

where $n$ is the number of CdTe-CdTe bonds of the particle $j, \Delta h \equiv h_{j^{\prime}}-h_{j}+1$ is the difference between the initial and final height of the particle, and $p \in(0,1)$ is a parameter.

3. Revaporization. The revaporization, which occurs at a rate $R_{3}$, consists of the exclusion of the highest deposited CdTe adatom in a randomly chosen column. Similarly to the diffusion, the revaporization rate is given by a Arrhenius-like law

$$
R_{3}=\frac{d^{*} k_{B} T}{h} \exp \left(-E_{v a p} / k_{B} T\right),
$$

where $E_{\text {vap }}$ is the effective energy for the revaporization. Again, this process will be implemented or not with probability $q^{n}$, where the parameter $q \in(0,1)$. For sake of simplicity, we used $q=p=0.1$ in all simulations.
The simulation procedure is the following. At each time step, one of the three processes deposition, diffusion, or revaporization is selected and, if the case, implemented with probabilities $P_{1}, P_{2}$, and $P_{3}$, respectively, and the time is incremented by $\Delta t=1 / L$. These probabilities are normalized and proportional to the correspondent rate, i. e., are given by

$$
P_{i}=\frac{R_{i}}{\sum_{j=1}^{3} R_{j}} .
$$

\section{RESULTS}

In all simulations, we considered temperatures corresponding to those of the experiments, namely, $200-400{ }^{\circ} \mathrm{C}$. The experimental device provides a constant deposition rate $R_{1}=$ $0.32 \mathrm{ML} / \mathrm{min}$. It was observed that no CdTe remains in the substrate for temperatures higher than $400{ }^{\circ} \mathrm{C}$. Considering this observation, one can estimate the activation energy for the revaporization. Using this experimental evidence and that material must remain on the substrate for $T \leq 300^{\circ} \mathrm{C}$ (Fig. 1), we estimated $E_{\text {vap }}$ in the interval $1.60-1.70 \mathrm{eV}$. Precise informations about the activation energies for the diffusion are not available, but we expect $E_{d i f}<E_{\text {vap }}$.

The effect of temperature is illustrated in Fig. 3, in which $T$ was varied in the interval $200-380^{\circ} \mathrm{C}$. Notice that for the higher temperature $\left(T=380{ }^{\circ} \mathrm{C}\right)$, desorption becomes dominant in quantitative agreement with the experiments. For $T \geq 250{ }^{\circ} \mathrm{C}$, the profiles exhibit QDs that apparently increase in number and decrease in width as the temperature increases. This is a typical feature of the VW growth mode observed in CdTe/Si (Fig. 1) and similar systems [12].

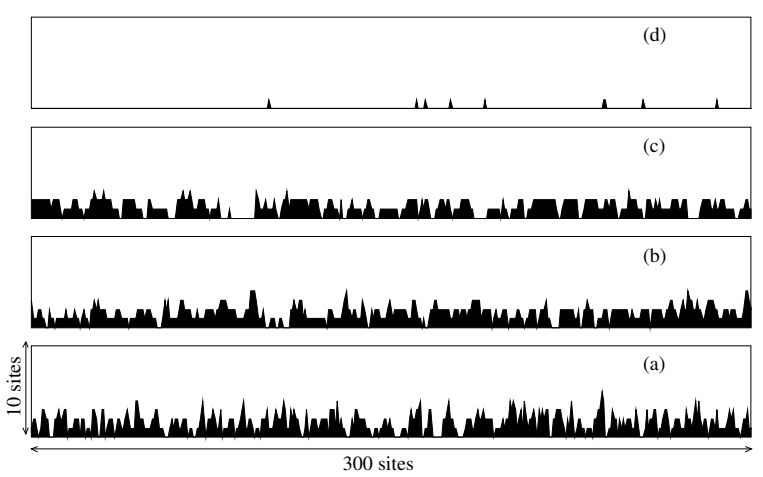

FIG.3. Effects of temperature on the interfaces with 1.6 ML of evaporated CdTe. The temperatures used were (a) $T=200{ }^{\circ} \mathrm{C}$, (b) $T=250{ }^{\circ} \mathrm{C}$, (c) $T=300{ }^{\circ} \mathrm{C}$, and (d) $T=380{ }^{\circ} \mathrm{C}$, while the other parameters were $p=q=0.1, E_{\text {vap }}=1.65 \mathrm{eV}$, and $E_{\text {dif }}=1.3 \mathrm{eV}$.

The height and width distributions of the QDs were evaluated for distinct temperatures. In order to determine the QD heights and width, each dot was fitted by a quadratic polynomial null at both of its extremities. The heights are assumed as the curve maximum. In Fig. 4, the distributions obtained using this method are drawn. As in the experiments, the height 
distributions were fitted by two-pick Gaussian curves. As the temperature increases, the curves become sharper and the correspondent peaks shift to smaller heights. In other words, the number of small QDs increases while the number of the larger ones decreases. This qualitative behavior was observed for $\mathrm{CdTe} / \mathrm{Si}$ system. The QD width distributions have a similar qualitative behavior at higher temperatures (figure 4(b)), but the data are poorly fitted by two-peak Gaussian curves. Indeed, after the initial increase, the distributions decay exponentially for large width values, as can be seen in the inset of Fig. 4(b). The same asymptotic decay was found for the height distributions.
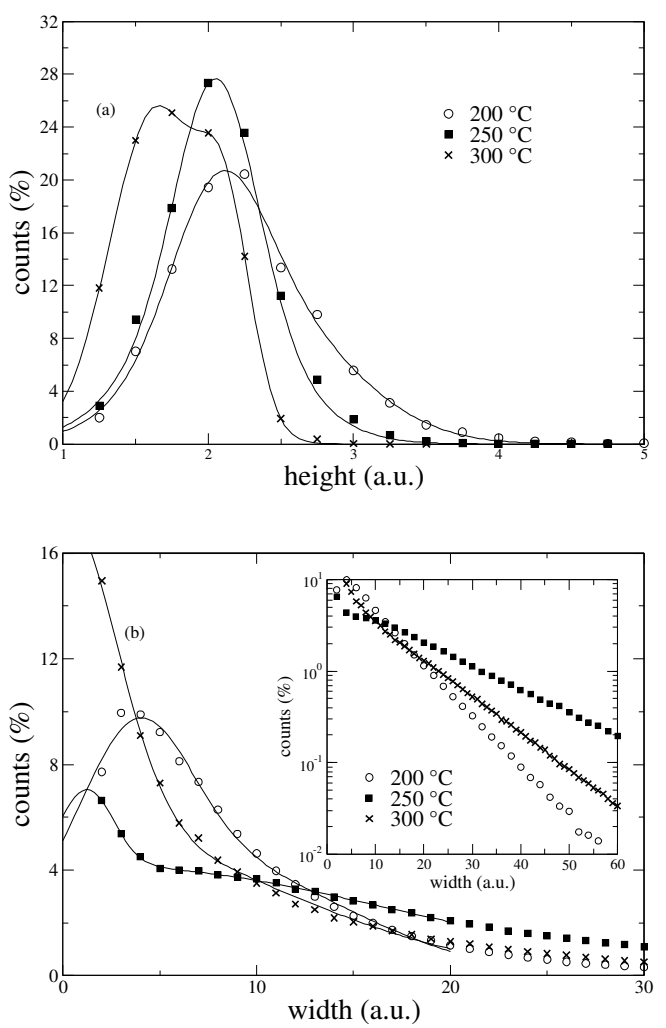

FIG.4. (a) Height and (b) width distributions for distinct temperatures. In inset, width distributions are drawn in semi-log plots becoming neat the asymptotically exponential decays. The solid lines correspond to the nonlinear fits. 1.6 ML of evaporated material, $P_{\text {vap }}=1.65 \mathrm{eV}, P_{\text {dif }=1.3} \mathrm{eV}$, and $p=q=0.1$ were used in all curves.

An important aspect observed in the width distributions is the curve inversion as the temperature increases (Fig. 4(b)). Comparing the distributions for $T=200^{\circ} \mathrm{C}$ and $T=250^{\circ} \mathrm{C}$, one can see that the first curve is sharper than the second one. But, comparing the distributions for $T=250{ }^{\circ} \mathrm{C}$ and $T=300{ }^{\circ} \mathrm{C}$, the opposite is observed. This suggests a transition from SK to VW growth mode. Rigorous simulations show the QD width increases in the low temperature regime, a signature of the SK growth mode, and decreases for the high ones, a characteristic of the VW growth mode. In CdTe/Si experiments only the decreasing regime of the QD mean width was observed. However, the mean height is always a decreasing function of temperature in agreement with VW mode. Additional experiments at lower temperatures should be executed in order to verify if SK-like growth is also present in $\mathrm{CdTe} / \mathrm{Si}$ systems. Notice that these data quantitatively disagree with the experiments because the growing mean width regimes were not experimentally observed in the interval $200-300{ }^{\circ} \mathrm{C}$ (Fig. 1). Indeed, is not surprising a quantitative disagreement between experiment and simulations since we used a $1 \mathrm{~d}$ model to study a $2 \mathrm{~d}$ system.

\section{CONCLUSIONS}

In the present work we studied a one dimensional model for the growth of CdTe quantum dots (QDs) on Si substrates. The experimental system follows the Volmer-Weber (VW) growth mode, with nucleation of CdTe dots on the Si surface [8] for less than one monolayer of deposited material. AFM analysis of the samples showed that the size and density of QDs can be controlled by properly adjusting the growth temperature. These quantities exhibit opposite behavior when compared to that (behavior) observed in systems following StranskiKrastanow (SK) growth mode used in almost the totality of the growth of semiconductor nanostructures. In the present model, CdTe adatoms are deposited on a one-dimensional $\mathrm{Si}$ substrate. The model includes also thermally activated diffusion e revaporization of CdTe atoms. The central feature of the model is the differences among the diffusion and evaporation rules for CdTe particles on Si substrate and CdTe particles on other previously deposited CdTe layers.

Even this over-simplified one-dimension model revealed several fundamental features present in the experiments. In the range of temperature corresponding to that of the experiments, the patterns exhibit island nucleation with width and height distributions in qualitative agreement with those observed in the experiments. The main model ingredient that originates the QDs is the difference between diffusion and revaporization rules for $\mathrm{CdTe}$ on the initial $\mathrm{Si}$ substrate and CdTe on the other previously deposited CdTe particles. For high temperatures, the QD size distributions shift to smaller sizes and the number of smaller dots increases while the number of larger ones decreases, a feature which agrees with the experimental observations and has been assigned as a characteristics of the VW growth mode. However, the model predicts an inversion of this behavior for lower temperatures, a feature which is a characteristic of the SK growth mode. Such a transition from VW to SK growth mode at low temperatures has not been observed experimentally. At the light of the model, the origin of this transition lies on the following: For lower temperatures, a particle on the Si substrate diffuses for a long time before its revaporization, resulting in an increasing chance to reach and aggregate to a previously deposited island. The result is the layer by layer (SK) growth mode. However, for larger temperatures, the particle evaporates shortly and, consequently, only those deposited near to 
or on a CdTe island have a significant probability to remain on the substrate, leading to the VW growth mode.

\section{Acknowledgments}

This work was supported by the CNPq, FAPEMIG, FACEPE, and FINEP Brazilian agencies.
[1] L. Goldstein, F. Glas, J. Y. Marzin, M. N. Charasse, and G. Le Roux, Appl. Phys. Lett. 47, 1099 (1985).

[2] D. J. Eaglesham and M. Cerullo, Phys. Rev. Lett. 64, 1943 (1990).

[3] M. Pinczolitz, G. Springholz, and G. Bauer, Appl. Phys. Lett. 73, 250 (1998).

[4] F. Widmann, B. Daudin, G. Feuillet, Y. Samson, J. L. Rouvière, and N. Pelekanos, J. Appl. Phys. 83, 7618 (1998).

[5] S. H. Xin, P. D. Wang, A. Yin, C. Kim, M. Dobrowolska, J. L. Merz, and J. K. Furdyna, Appl. Phys. Lett. 69, 3884 (1996).

[6] Y. Terai, S. Kuroda, K. Takita, T. Okuno, and Y. Masumoto, Appl. Phys. Lett. 73, 3757 (1998).

[7] S. Kuroda, Y. Terai, K. Takita, T. Okuno, and Y. Masumoto, J.
Cryst. Growth 184/185, 274 (1998)

[8] S. O. Ferreira, E. C. Paiva, G. N. Fontes, and B. R. A. Neves, J. Appl. Phys. 93, 1195 (2003).

[9] P. Chen, Q. Xie, A. Madhukar, L. Chen, and A. Konkar, J. Vac. Sci. Technol. B 12, 2568 (1994).

[10] G. Abstreiter, P. Shittenhelm, C. Engel, E. Silveira, A. Zrenner, Semicond. Sci. Technol. 11, 1521 (1996).

[11] M. Sopanen, H. Lipsanen, and J. Aholpeto, Appl. Phys. Lett. 67, 3768 (1995).

[12] S.O. Ferreira, B.R.A. Neves, R. Magalhães-Paniago, A. Malachias, P.H.O. Rappl, A.Y. Ueta, E. Abramof, and M.S. Andrade, J. Cryst. Growth 231, 121 (2001). 